\title{
A UWB imaging system to detect early breast cancer in heterogeneous breast phantom
}

\begin{abstract}
This paper presents an experimental early breast cancer detection system in terms of heterogeneous breast phantom. The system consists of commercial Ultrawide-Band (UWB) transceivers and our developed Neural Network (NN) based Pattern Recognition (PR) software for imaging. A simple way to construct cancer- tissue and heterogeneous breast phantom using available low cost materials and their mixtures is also proposed here. The materials are: (i) A mixture of petroleum jelly, soy oil, wheat flour and water as heterogeneous tissue; (ii) A particular glass as skin; and (iii) A specific mixture of water and wheat flour as cancer- tissue. All the materials and their mixtures are considered according to the ratio of the dielectric properties of the breast tissues. To experimentally detect cancer, the UWB signals are transmitted from one side of the breast phantom and received from opposite side diagonally. By using discrete cosine transform (DCT) of the received signals, a Neural Network (NN) is trained, tested and interfaced with the UWB transceiver to form the complete system. The achieved detection rate of cancer cell's existence, size and location are approximately $100 \%, 93.1 \%$ and $93.3 \%$ respectively.
\end{abstract}

Keyword: Breast phantom for UWB imaging; Early breast cancer detection; Neural network 